\title{
La sequía en España: análisis del fenómeno a través del estudio de las noticias de prensa recogidas por Hispagua durante el año 2005
}

\author{
Elena Fernández Sánchez \\ Sonia Jiménez Hidalgo \\ Javier Salvador Bruna \\ Centro de Información y Documentación Científica del CSIC
}

\section{Resumen}

El servicio de información Hispagua recoge una selección de las noticias que tratan sobre el agua. En este artículo se estudian aquellas correspondientes al año 2005 sobre el problema de la sequía, en todos sus aspectos. Se ha realizado el análisis de los organismos e instituciones con mayor protagonismo en dicho tema, así como la distribución de las noticias atendiendo a su procedencia geográfica y a su temática, con especial seguimiento de las informaciones de carácter político que se sucedieron durante el periodo analizado.

Palabras clave: Análisis bibliométrico. Prensa diaria. Sequía. Hispagua. Portales científicos. España.

\section{Summary}

This paper analizes the 2005 press news related to the drought in Spain, obtained in a database included in the Hispagua information service. The more prolific institutions, as well as the distribution of the press news in relation with their geographical origin and the topics treated, taking special interest in political information, have been studied.

Key words: Biblometric analysis. Daily news. Drought. Spain. Scientific portals. Hispagua.

\section{Introducción}

El año hidrometeorológico que comenzó el 1 de septiembre de 2004 y terminó el 31 de agosto de 2005 pasará a la historia de la climatología por ser el más seco de la historia de España desde que se empezó a hacer mediciones sistematizadas de las precipitaciones, es decir, desde 1947, según informa el Instituto Nacional de Meteorología (INM). Durante aquel año, la precipitación media fue, para el 
conjunto del territorio nacional, de 411 milímetros, lo que supone casi un $40 \%$ menos que el valor medio normal.

La sequía ha afectado, en mayor o menor medida, a la práctica totalidad de las regiones, salvo a Canarias, aunque el déficit mayor de precipitaciones fue el registrado en Extremadura, seguido por los de Andalucía, Castilla - La Mancha y Madrid. En estas regiones, junto con algunas zonas de Cataluña y del sur de Castilla y León, las precipitaciones acumuladas no han llegado ni siquiera al 50\% de los valores normales. En zonas del oeste de Andalucía, del Bajo Guadalquivir y del sureste de Castilla - La Mancha se han registrado totales pluviométricos acumulados que apenas superan el 35\% de los valores medios. En el resto de las regiones se aprecia también un déficit de precipitaciones, aunque en este caso de manera más moderada, de entre el $60 \%$ y el $75 \%$ de los valores medios. También Portugal está sufriendo un fenómeno análogo al español, así como la parte oeste de Francia, donde se están alcanzando los valores más bajos en sus reservas de agua desde 1976.

Con independencia de fenómenos críticos que se puedan producir periódicamente, España es un país esencialmente seco y, junto con Malta, Italia y Chipre, padece el mayor estrés hídrico de la Europa de los 25.

Durante la pasada década, los países de Europa Central y algunos del oeste central europeo disminuyeron las extracciones de agua destinadas a la agricultura, a usos industriales y al consumo urbano. En cambio, en los países del suroeste se produjo un incremento del agua destinada al sector agrícola, si bien en esta misma zona se redujo el consumo dedicado a la producción de energía (El agua en Europa, 2003).

El impacto que la escasez de agua tiene sobre nuestro país, y que aflora en toda su magnitud en épocas de intensa sequía, afecta a todos los sectores esenciales de nuestra economía aunque con diferentes grados de inmediatez o urgencia. En el caso de los sectores agrícola y ganadero el impacto se percibe de forma inmediata, ya que si no se dispone de agua para riego se pone en peligro la cosecha de todo un año y los pastos destinados a la ganadería. En otros sectores, como el turismo, la construcción, y la producción de energía hidroeléctrica, las consecuencias no se dejan notar de manera tan inmediata, ya que siempre queda la esperanza de abundantes lluvias en un futuro más o menos próximo.

\section{Gestión del agua en España}

La gestión del agua en España presenta una característica muy singular. Esta singularidad radica en la gran complejidad del reparto entre las diferentes administraciones de las responsabilidades en la gestión del agua. Así, por ejemplo, las competencias sobre abastecimiento urbano se encuentran distribuidas entre las siguientes instituciones de carácter público: a) Ministerio de Medio Ambiente, $b$ ) Ministerio de Industria, Turismo y Comercio, c) Ministerio de Sanidad y Consumo, 
d) Confederaciones Hidrográficas, e) Consejerías de Obras Públicas (administraciones autonómicas), $f$ ) Consejerías de Sanidad (administraciones autonómicas), g) Consejerías de Industria y sus comisiones de precios (administraciones autonómicas).

El resultado final de tal atomización de responsabilidades, dentro de un organigrama tan complejo, es la dificultad para la toma de decisiones que, en muchos casos, conllevan objetivos vinculados a diversos intereses y cuyo resultado es el enfrentamiento entre los diferentes actores y los propios ciudadanos, situación que podríamos considerar como "la guerra del agua", y que obviamente se acentúa en periodos críticos como es la época de sequía que padecemos (García-Serra y Cabrera, s. f.).

Las consecuencias de la sequía en los diferentes ámbitos sociales también pueden extrapolarse a las medidas de prevención de la misma, que abarcan no solo a las infraestructuras hídricas (por lo demás, muy variadas, como veremos al analizar las noticias de prensa), sino que también adquieren dimensiones sociales, especialmente en lo que se refiere a las campañas de concienciación para lograr un uso racional del agua. Por supuesto, y de manera significativa, la política hidráulica vuelve a ser fundamental para la prevención de los daños de la sequía.

Como hemos visto en este breve repaso, la sequía incide en el desarrollo económico, social y político de un país, y además se trata de un fenómeno difícilmente reducible a unos pocos indicadores meteorológicos o económicos. Son tantas sus derivaciones, y es tan cercano el impacto que sus consecuencias tienen para el conjunto de los ciudadanos, que sin duda merece la pena acercarse al fenómeno de la sequía desde una perspectiva social.

\section{Objetivos}

Este estudio pretende contribuir a la comprensión de la sequía desde un enfoque social, a través del impacto informativo de este fenómeno tal y como queda expresado en los medios de comunicación y, más en concreto, en la prensa escrita, tanto regional como nacional.

Se ha considerado que la prensa escrita es un medio capaz de abarcar todos los aspectos relativos a la sequía que han sido descritos brevemente en la introducción a este trabajo, ya que, en su mayor parte, los diarios escogidos son tanto de carácter nacional como procedentes de las diferentes comunidades autónomas españolas.

\section{Metodología}

Las noticias de prensa analizadas corresponden a las recogidas en una de las bases de datos accesibles a través de Hispagua (SEMIDE, s. f.), un servicio de información desarrollado por el Centro de Información y Documentación Científica

Scire. 12 : 2 (jul.-dic. 2006) 137-150. ISSN 1135-3716. 
(CINDOC) del CSIC. El periodo analizado es el año 2005, y las noticias han sido extraídas de los principales periódicos españoles durante ese año.

\subsection{Selección de noticias}

El servicio de consulta de noticias de prensa ofrecido por Hispagua dispone de una base de datos donde se recoge una selección de noticias discriminadas de acuerdo con su interés informativo. Por tanto, no se han tenido en cuenta todas aquellas noticias reflejadas en la prensa durante el periodo analizado, sino un conjunto de las mismas suficientemente representativo, a nuestro entender, de la forma en que los medios de comunicación escritos han abordado el problema de la sequía. Se ha trabajado con un conjunto de 809 noticias de 47 medios diferentes (véase anexo 1), que comprenden tanto periódicos españoles como agencias de prensa. La práctica totalidad de las informaciones tienen como objeto la sequía en España, siendo muy escasas las noticias relativas a la sequía en otras zonas de Europa o en el resto del mundo.

\subsection{Tratamiento documental de las noticias analizadas}

El tratamiento documental de la información tomada como muestra contempla tres etapas:

a) La base de datos de noticias de Hispagua, cuyas referencias han sido enriquecidas con metadatos, con un tratamiento documental que incluye una serie de descriptores, topónimos e identificadores, ha servido para realizar una búsqueda a partir del descriptor sequía y con el límite temporal del año 2005. Los registros de esta búsqueda son los que forman la base de datos específica para este trabajo, a partir de la cual se ha realizado el estudio.

b) Se ha realizado una segunda indización más en profundidad de estas 809 noticias adoptando descriptores temáticos específicos. El número total de descriptores específicos para este trabajo ha sido de 56, distribuidos en 14 categorías (anexo 2). También se ha añadido un nuevo campo para describir la ideología política de la información de la noticia, en caso de que existiese.

c) Se han llevado a cabo las consultas necesarias sobre la nueva base de datos para obtener los resultados que se describen más adelante.

\section{Análisis de la información}

La muestra de noticias nos ha permitido realizar un análisis tomando como variables los siguientes elementos: distribución geográfica del asunto de la noticia, frecuencia de los indicadores utilizados en el tratamiento documental, frecuencia de las temáticas más comunes y análisis de los medios de comunicación representados. Veamos con detalle cada uno de ellos. 


\subsection{Distribución geográfica}

Andalucía ha sido la comunidad que ha generado mayor número de noticias (24\%), seguida por Cataluña y la Comunidad Valenciana (16\% ambas), y Murcia, que representa el (15\%). Los resultados reflejan que, aunque la sequía ha afectado a todo el país con la excepción de Canarias, son las comunidades históricamente más secas las más afectadas por este fenómeno, junto con Cataluña (véase figura 1).

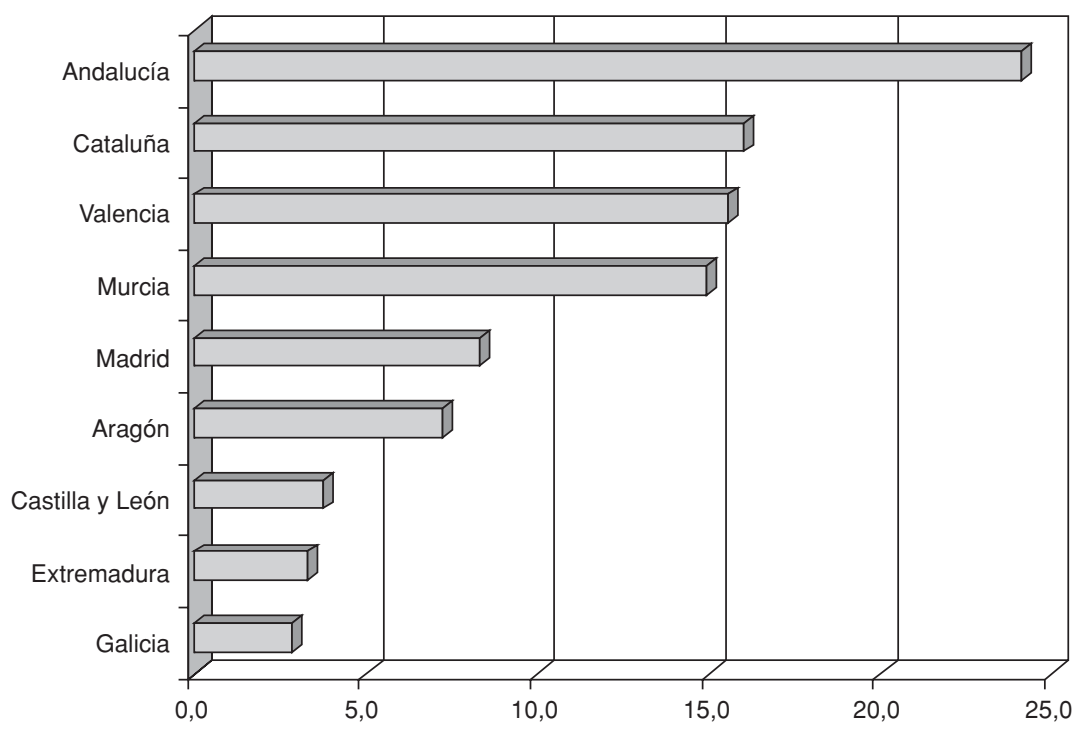

Figura 1. Distribución de noticias por área geográfica (\% respecto al total).

\subsection{Distribución de los identificadores}

El análisis de los identificadores utilizados permite concluir que la polémica del trasvase Tajo-Segura ha sido la noticia mejor reflejada en la prensa: un $27 \%$ de las noticias tenían que ver con dicho problema. En cuanto a las organizaciones, las más frecuentemente mencionadas han sido el Ministerio de Medio Ambiente $(25,2 \%)$ y las confederaciones hidrográficas del Segura y del Ebro $(11,8 \%$ y $9,2 \%$ respectivamente) (véase figura 2). Estos resultados identifican de forma clara los asuntos más conflictivos en temas de agua, en los que, cómo no, figuran las cuencas del Segura y del Ebro, afectadas de forma diferente por la política hidráulica adoptada por el Gobierno. 


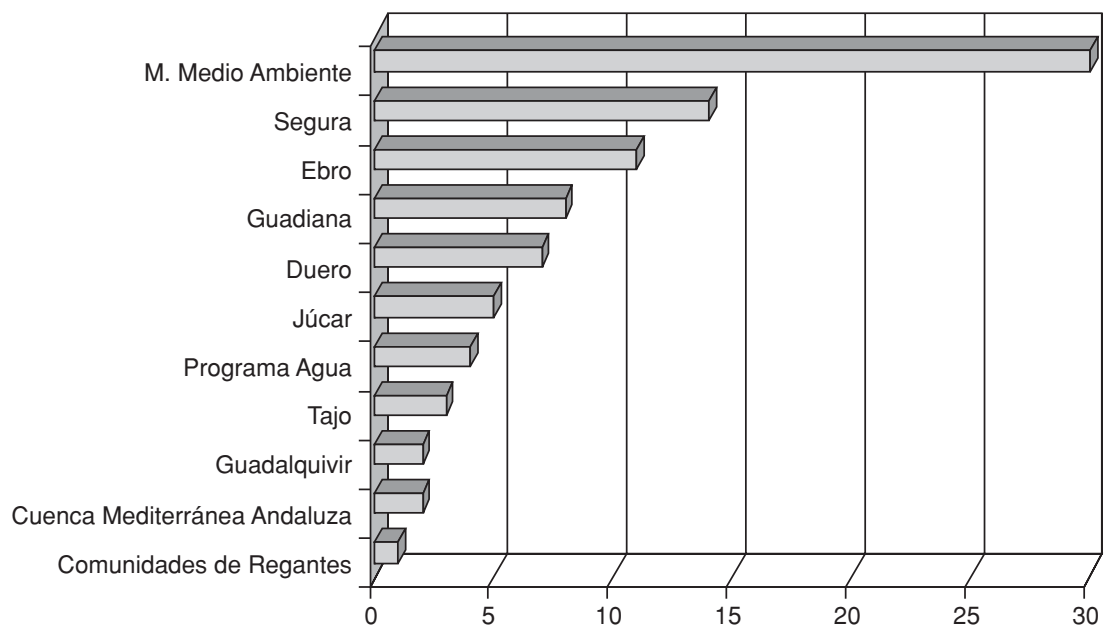

Figura 2. Distribución de noticias según identificadores (\% respecto al total).

\subsection{Frecuencia temática}

La tabla I presenta la distribución de las noticias por grandes apartados temáticos, de acuerdo con los bloques de descriptores utilizados para el análisis. El nú-

\begin{tabular}{|l|c|c|}
\hline Temática & N.o de descriptores & $\%$ \\
\hline Política & 310 & 19,99 \\
\hline Cultura del agua y consumo & 224 & 14,44 \\
\hline Sequía hidrológica & 205 & 13,22 \\
\hline Explotación de recursos hídricos & 194 & 12,51 \\
\hline Sequía agronómica & 162 & 10,44 \\
\hline Sequía meteorológica & 119 & 7,67 \\
\hline Economía & 104 & 6,71 \\
\hline Obras públicas e infraestructuras & 93 & 6,00 \\
\hline Medio ambiente & 60 & 3,87 \\
\hline Repercusiones sociales & 33 & 2,13 \\
\hline Sistemas de riego & 21 & 1,35 \\
\hline Calidad del agua & 11 & 0,71 \\
\hline Investigación & 8 & 0,52 \\
\hline Turismo & 7 & 0,45 \\
\hline
\end{tabular}

Tabla I. Distribución por temática.

Scire. 12 : 2 (jul.-dic. 2006) 137-150. ISSN 1135-3716. 
mero de descriptores es mayor que el número de noticias tomadas como referencia, ya que cada una de ellas, generalmente, posee más de un descriptor temático.

El análisis de cada uno de los apartados temáticos ha sido complementado con el análisis cronológico de las noticias, esto es, su distribución a lo largo del periodo anual considerado. Sin duda, al ser la sequía un fenómeno dependiente en gran parte de la meteorología, las variaciones de esta a lo largo del año inciden, como es lógico, en la expresión que los medios de comunicación hacen de ella.

\subsubsection{Información de carácter político}

Sin duda es la política hidráulica el tema más frecuente de todas las noticias analizadas, ya que representa un $20 \%$ respecto al total. En la figura 3 se puede observar que el primer máximo se alcanza durante mes de mayo y se mantiene de forma regular hasta los valores del mes de octubre.

Durante el primer trimestre del año, las decisiones políticas adoptadas son de carácter preventivo. Destacan algunas medidas del Ministerio de Medio Ambiente como la puesta en marcha de una red de indicadores globales de sequía (enero), licitaciones de urgencia en regiones sensibles a la sequía como las cuencas del Segura y del Duero y, en general, el sur peninsular, además de las peticiones a las diferentes confederaciones hidrográficas para que aceleren sus planes de emergencia ante la sequía. Por tanto, en esta primera parte, la Administración central se limitó a labores de prevención y coordinación de otros organismos. En cuanto a las comunidades autónomas, ya durante el mes de marzo se observan las primeras advertencias, sobre futuras restricciones, de momento tan solo en los sectores hidroeléctrico y agrícola, como en el caso de Cataluña. En Aragón y Galicia también destacan actuaciones, como la reducción de la extensión de tierra para cultivos y la puesta en marcha de obras de mejora del abastecimiento, respectivamente.

El segundo trimestre, de abril a junio, se inicia con los primeros decretos de la sequía, en este caso el aprobado por la Agencia Catalana del Agua. Desde el Gobierno central se da prioridad a las campañas de concienciación y, muy especialmente, a la iniciativa planteada desde el Ministerio de Medio Ambiente para informar de forma regular sobre la situación de la sequía. A este respecto, los mensajes emitidos por la ministra responsable de Medio Ambiente, y recogidos de forma semanal por los medios, se centran en dos aspectos básicos: las obras de emergencia para paliar los efectos de la sequía (tanto las ya realizadas como las previstas, entre las que destaca la anticipada puesta en marcha de la planta desalinizadora de San Pedro del Pinatar) y la ausencia de restricciones para consumo de agua de boca durante todo el periodo de sequía.

Durante el mes de mayo se producen dos situaciones polémicas: por un lado, las tensiones entre el Gobierno regional de la Comunidad de Madrid y el Gobierno central ante las medidas previstas para reducir el consumo de agua; por otro, la

Scire. $12: 2$ (jul.-dic. 2006) 137-150. ISSN 1135-3716. 
solicitud ante las diferentes instancias europeas para recabar ayudas para las zonas afectadas por la sequía, amparándose en su condición de regiones que han sufrido catástrofes climáticas.

Durante el mes de junio, las noticias sobre la sequía van incrementándose, incluyendo, además de los temas anteriores, otros asuntos nuevos. En especial, destaca la aprobación de un nuevo trasvase entre las cuencas del Tajo y del Segura, así como la aprobación por el Parlamento de un real decreto ley que aprueba ayudas económicas para el sector ganadero y agrícola. En cuanto a las comunidades autónomas, destaca el hecho de que Cataluña mantenga su "decreto de sequía", así como las medidas puestas en marcha por la Junta de Andalucía.

El tercer trimestre (julio, agosto y septiembre) se inicia con una de las noticias que más repercusión ha tenido en la prensa: la autorización por el Consejo de Ministros de 1 de julio de 2005 al trasvase de 82 hectómetros cúbicos del río Tajo al Segura. Las abundantes reacciones de los gobiernos autonómicos, así como la propia entidad de la noticia, hicieron que fuera la de mayor repercusión hasta ese momento del año. Además, durante ese mismo mes de julio otros dos asuntos más merecieron especial atención: en primer lugar, las medidas de restricción aplicadas en ciertas regiones, especialmente Andalucía y Madrid, acompañadas generalmente de las subsiguientes campañas de concienciación para uso racional del agua; en segundo lugar, la aprobación de protocolos de sequía en las confederaciones hidrográficas del Norte, el Tajo, el Ebro y el Guadiana, así como en la Mancomunidad de Regantes del Taibilla. Durante el mes de agosto se suceden las medidas de todas las administraciones para reducir el consumo de agua, siendo especialmente reseñadas las acometidas por el Ayuntamiento de Madrid. No obstante, y en un periodo donde decrece gradualmente el agua embalsada, las informaciones más destacadas son la creación del Observatorio de la Sequía y las primeras propuestas de la Agencia Catalana de Agua, que estudiaba en ese momento posibles recortes al consumo humano. En septiembre, pese a las lluvias, el Ministerio de Medio Ambiente continúa expresando la necesidad de realizar nuevas obras, mientras se recurre a la apertura de pozos en algunas zonas como la Comunidad de Madrid. Finalmente, el trimestre (y el año hidrológico) se cierra con la polémica generada por las discusiones ante un nuevo trasvase Tajo-Segura.

Los últimos meses del año quedan marcados por la aprobación definitiva de un nuevo trasvase de agua desde la cabecera del Tajo a la cuenca del Segura. El Ministerio de Medio Ambiente asegura que el consumo humano está garantizado en dicha zona, a pesar de lo cual anuncia concesiones económicas para el inicio de obras de emergencia. Las protestas de Murcia, que no recibió ningún aporte del trasvase, son así contestadas por los responsables del Gobierno central. Además de este nuevo episodio de trasvase, la medida más significativa fue la aprobación por parte del Consejo de Ministros del real decreto que permitía medidas extraordinarias para 
las cuencas del Segura, el Júcar y el Tajo. Dicho decreto cedía a las confederaciones hidrográficas la potestad para regular, en situaciones excepcionales, los usos del agua, la apertura de pozos de sequía y la aplicación de restricciones. Poco después, las cuencas del Ebro, el Guadiana y el Guadalquivir también fueron objeto de la atención del Ministerio de Medio Ambiente, que aprobó un decreto similar al puesto en marcha en el sur peninsular.

En el siguiente esquema se puede observar, de forma sintetizada, la principal evolución de los asuntos tratados a lo largo del tiempo:
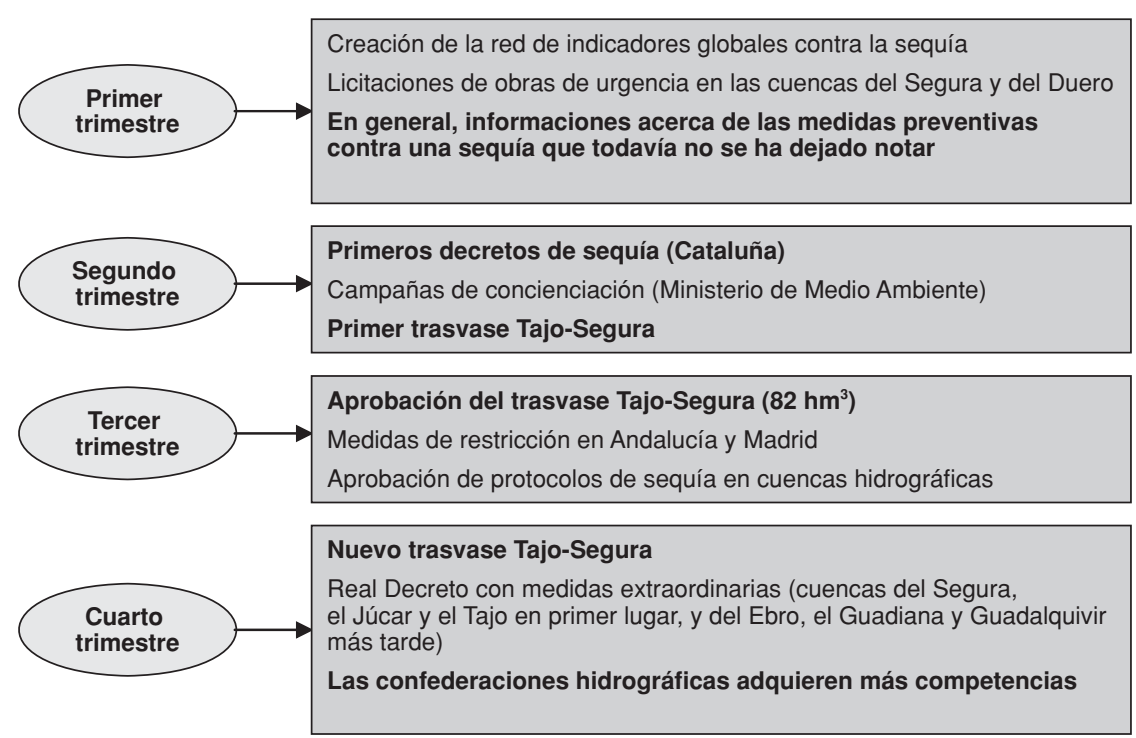

\subsubsection{Otras informaciones}

El segundo tema en importancia tratado por la prensa fue la cultura del agua y el consumo, con un 14\%. Al igual que en el caso anterior, el incremento importante de número de noticias empieza en el mes de mayo, y se mantiene hasta septiembre. La información recogida en este apartado está relacionada fundamentalmente con campañas de concienciación de ahorro de agua y consumo responsable.

La sequía hidrológica y la explotación de recursos hídricos son otros dos asuntos frecuentemente reseñados; de acuerdo con nuestro estudio, ambos son protagonistas del $13 \%$ de las noticias. Su evolución cronológica es similar, ya que reflejan dos aspectos íntimamente relacionados: comienzan su ascenso en el mes de mayo hasta llegar a un máximo en septiembre.

Scire. $12: 2$ (jul.-dic. 2006) 137-150. ISSN 1135-3716. 
El resto de los aspectos tratados presentan valores inferiores a los ya citados, y su distribución se puede observar en la figura 3.

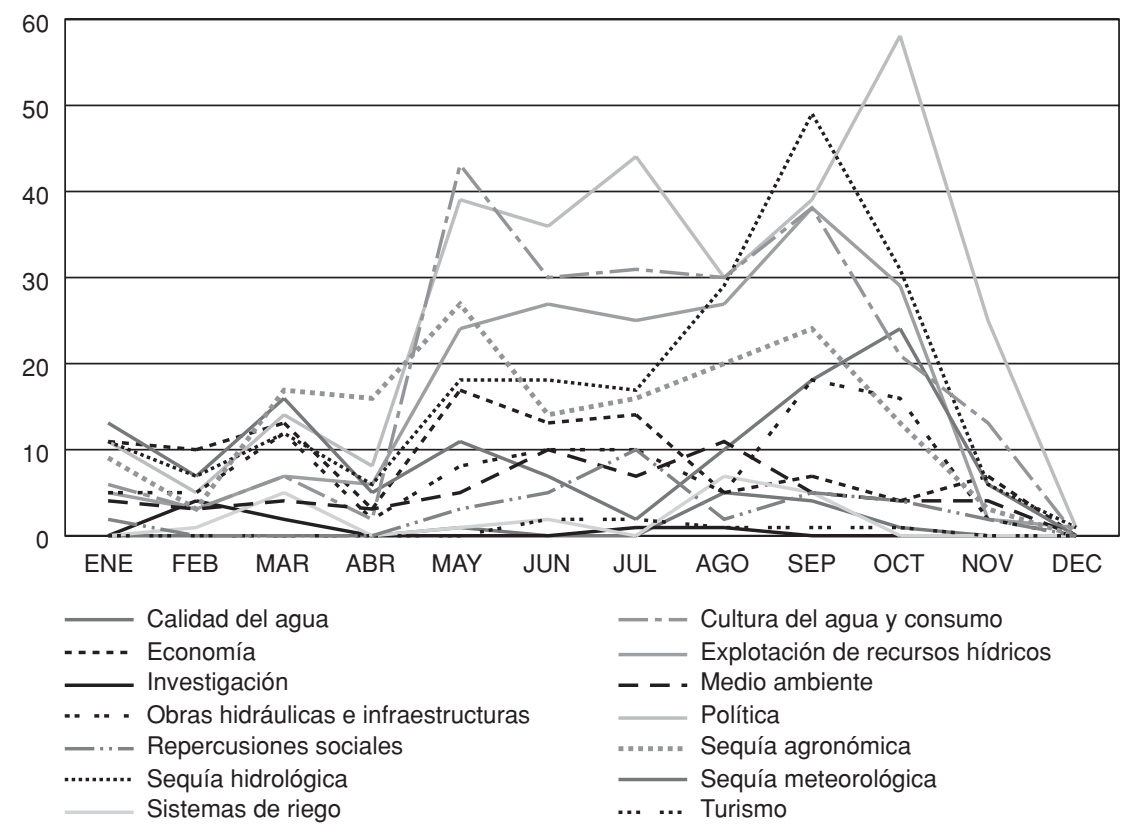

Figura 3. Distribución de temáticas por meses.

\subsection{Medios de comunicación}

Aproximadamente la mitad de las noticias analizadas correspondieron a medios y agencias de cobertura nacional, como puede observarse en la tabla II. Así, Europa Press, El País, ABC y EFE reúnen aproximadamente el 50\% de las noticias analizadas.

De manera aproximada, el reparto del número de noticias es equilibrado según el tipo de organismo que las emite: un $32 \%$ corresponde a agencias de noticias, un $30 \%$ a diarios de tirada nacional y un $38 \%$ a prensa regional o local.

\section{Conclusiones}

Las noticias de prensa nacional y regional constituyen una herramienta importante para conocer los acontecimientos ocurridos en un país o región geográfica en determinados periodos de tiempo. Este trabajo se ha centrado en la

Scire. 12 : 2 (jul.-dic. 2006) 137-150. ISSN 1135-3716. 


\begin{tabular}{|l|r|c|}
\hline Medio & N.o de artí:ulos & $\%$ \\
\hline Europa Press & 183 & 22,79 \\
\hline El País & 77 & 9,59 \\
\hline ABC & 73 & 9,09 \\
\hline EFE & 72 & 8,97 \\
\hline La Verdad & 54 & 6,72 \\
\hline El Mundo & 30 & 3,74 \\
\hline Levante & 27 & 3,36 \\
\hline Información & 21 & 2,62 \\
\hline Heraldo de Aragón & 19 & 2,37 \\
\hline La Vanguardia & 18 & 2,24 \\
\hline Las Provincias & 18 & 2,24 \\
\hline Estrella Digital & 16 & 1,99 \\
\hline La Voz de Galicia & 16 & 1,99 \\
\hline El Periódico de Aragón & 13 & 1,62 \\
\hline Sur & 13 & 1,62 \\
\hline Expansión & 12 & 1,49 \\
\hline La Opinión de Murcia & 11 & 1,37 \\
\hline La Razón & 11 & 1,37 \\
\hline El Periódico & 10 & 1,25 \\
\hline Otros & 109 & \\
\hline & & 13,57 \\
\hline
\end{tabular}

Tabla II. Distribución de las noticias analizadas según los medios.

temática del agua, y concretamente en la sequía, pero podría extrapolarse para ser usado en cualquier disciplina o acontecimiento.

El estudio también pone de manifiesto que la utilidad de esta fuente de información se basa en la recopilación de las noticias, previamente sometidas a un análisis documental, creando bases de datos. La razón es evidente: a través de un cuidadoso análisis documental de las noticias se optimizan las operaciones de búsqueda y aumenta la accesibilidad para los usuarios e investigadores que necesiten acceder a esta información.

Las noticias de prensa se configuran en un medio que permite conocer la memoria histórica, mostrando los temas estrella y sus acontecimientos relacionados de una forma ajustada a la realidad. Así, en este estudio se ha demostrado que las zonas geográficas que más noticias protagonizan son aquellas que más sufren la sequía, como cabía esperar. De modo similar, es la información de carácter político la más importante, ya que su repercusión es muy general e inmediata.

Scire. 12 : 2 (jul.-dic. 2006) 137-150. ISSN 1135-3716. 
Por último, el hecho de que las noticias de prensa se encuentren accesibles de forma gratuita en portales especializados en una disciplina, como es el caso de Hispagua, pone una vez más de manifiesto el importante servicio que estos sistemas de información proporcionan a toda la sociedad.

\section{Referencias}

El agua en Europa: una evaluación basada en indicadores (2003). Resumen. http://reports. es.eea.europa.eu/report_2003_0617_150910/es/ES_WIR_WWW.pdf (2006-01-23).

García-Serra, Jorge; Cabrera, Enrique (s. f.). Las estructuras de gestión del agua en España y en Israel: dos modelos contrapuestos. http://www.us.es/ciberico/archivos_acrobat/ zaraponen6cabrera.pdf (2006-01-23).

SEMIDE. Hispagua: Sistema Español de Información sobre el Agua (s. f.). http://hispagua. cedex.es (2006-01-23).

\section{Anexo 1. Fuentes de información}

\section{Periódicos de tirada nacional}

El País

$A B C$

El Mundo

La Vanguardia

La Razón

Estrella Digital

Cinco Días

Expansión

\section{Periódicos regionales}

Córdoba

Correo Gallego

Diari Segre

Diario de Cádiz.

Diario de Ibiza

Diario de Jerez

Diario de León

Diario de Mallorca

Diario de Navarra

Diario de Noticias

Diario de Sevilla

Diario de Tarragona

Diario del Altoaragón

Diario Vasco

El Adelanto

El Comercio

El Correo 
El Día de Córdoba

El Diario Montañés

El Norte de Castilla

El Periódico

El Periódico de Aragón

El Periódico de Extremadura

Europa Sur

Heraldo de Aragón

Hoy

Ideal

Información

La Opinión de Málaga

La Opinión de Murcia

La Opinión - El Correo de Zamora

La Rioja

La Verdad

La Voz de Galicia

Las Provincias

Levante

Mediterráneo

Sur

\section{Agencias de prensa}

Europa Press

EFE

Anexo 2. Descriptores temáticos utilizados

\begin{tabular}{|l|l|}
\hline Explotación de recursos hídricos & Pozos \\
& Desalación \\
& Sobreexplotación \\
& Trasvases \\
& Agua de lluvia \\
& Aguas subterráneas \\
& Uso de aguas depuradas \\
& Energía \\
\hline Sequía hidrológica & Caudal de ríos \\
& Nivel de embalses \\
& Nivel de acuíferos \\
& Nivel de lagos \\
\hline Medio ambiente & Efectos de la sequía en flora y fauna \\
& Efectos de la sequía en ríos y cauces \\
& Efectos generales en el medio ambiente \\
& Efectos sobre los humedales \\
& Incendios forestales \\
& Cambio climático \\
\hline
\end{tabular}

Scire. 12 : 2 (jul.-dic. 2006) 137-150. ISSN 1135-3716. 


\begin{tabular}{|c|c|}
\hline Política & $\begin{array}{l}\text { Medidas del Gobierno central } \\
\text { Medidas de administraciones autonómicas } \\
\text { Medidas de administraciones locales } \\
\text { Medidas comunitarias } \\
\text { Medidas de confederaciones hidrográficas } \\
\text { Distribución de competencias }\end{array}$ \\
\hline Sistemas de riego & $\begin{array}{l}\text { Modernización } \\
\text { Modernización: riego por goteo } \\
\text { Modernización: riego por aspersión } \\
\text { Modernización: otros sistemas de riego } \\
\text { Modernización: prevención de fugas }\end{array}$ \\
\hline Cultura del agua y consumo & $\begin{array}{l}\text { Campañas de concienciación } \\
\text { Consumo } \\
\text { Uso racionales del agua } \\
\text { Restricciones consumo humano } \\
\text { Escasez de agua }\end{array}$ \\
\hline Economía & $\begin{array}{l}\text { Precio del agua } \\
\text { Repercusiones económicas de la sequía } \\
\text { Compensaciones económicas } \\
\text { Ayudas para la prevención } \\
\text { Medidas para prevenir la sequía }\end{array}$ \\
\hline Sequía meteorológica & $\begin{array}{l}\text { Datos de pluviosidad } \\
\text { Otros datos }\end{array}$ \\
\hline Obras hidráulicas e infraestructuras & $\begin{array}{l}\text { Depuradoras } \\
\text { Desalinizadoras } \\
\text { Presas y embalses } \\
\text { Obras de trasvase } \\
\text { Abastecimiento } \\
\text { Saneamiento }\end{array}$ \\
\hline Sequía agronómica & $\begin{array}{l}\text { Pérdida de cultivos } \\
\text { Otros efectos sobre los cultivos } \\
\text { Restricciones para uso agrícola } \\
\text { Planes agrarios contra la sequía }\end{array}$ \\
\hline Turismo & Efectos sobre el turismo \\
\hline Repercusiones sociales & Protestas y manifestaciones \\
\hline Investigación & Investigación \\
\hline Calidad del agua & Calidad del agua \\
\hline
\end{tabular}

